Computer Optics and Nanophotonics

\title{
PROFESSOR S.V. KARPEEV IS 60 YEARS OLD
}

\author{
E.I. Kolomiets
}

Samara National Research University, Samara, Russia

\begin{abstract}
The article presents a summary of scientific and pedagogical activity of a leading researcher of the Institute of Image Processing Systems of Russian Academy of Sciences, Doctor of Physical and Mathematical Sciences, Professor Sergey Vladimirovich Karpeev, a well known expert in the field of computer optics. The celebrant's contribution to the development of methods of research of diffractive optical elements and the use of computer optics methods to solve a wide range of problems is being analyzed in the article.
\end{abstract}

Keywords: computer diffractive optics, focusators, compensators, modans, photonic crystals, polarization-mode converters.

Citation: Kolomiets EI. Professor S.V. Karpeev is 60 years old. CEUR Workshop Proceedings, 2016; 1638: 217-225. DOI: 10.18287/1613-0073-2016-1638217-225

\section{Introduction}

In May, 2016 a scientist, a teacher, an expert in the field of research and development of diffractive optical elements, a leading researcher of Image Processing Systems Institute of Russian Academy of Sciences (IPSI RAS), a Professor of Samara nanoengineering Chair of Samara National Research University named after Academician Sergey Korolev, Doctor of Physical and Mathematical Sciences, Professor Sergey Karpeev has celebrated his jubilee.

The article presents a summary of scientific and pedagogical achievements of the celebrant, analyzes the contribution of Sergey Karpeev in the development of computer optics and optical studies.

\section{KuAI-SSAU}

In September, 1973 Sergey Karpeev became a student of the newly established Faculty of System Engineering of the Kuibyshev Aviation Institute (from 1992 Kuibyshev Aviation Institute (KuAI) was named Samara State Aerospace University (SSAU), and from 2015 - Samara National Research University). From the second course of his study at the institute Sergey Karpeev began to do research under the guidance of Victor Soifer, who at the time was the first dean of the faculty. That was when Soifer`s research interests have spread to a new and rapidly developing field of digi- 
tal holography, which later transformed into a more common one called computer optics. The Institute of Automation and Electrometry (IAE) then became one of the leaders in the new direction of the optical information science. It was in collaboration with researchers from Akademgorodok that the first studies on digital holography were held by the Soifer group. During his study Sergey Karpeev has twice passed practice in the Institute of Automation and Electrometry under the leadership of E. Nezhevenko, he met a young specialist A. Poleshchuk, and their cooperation continues to this day. At that time the USSR industry did not produce laboratory equipment for the coherent-optical research, so the first optomechanics set was made to order at the KuAI experimental plant according to the design made in Novosibirsk. Sergey Karpeev was directly involved in production, combining his studies with work at the Chair of Automated Control Systems (ACS) in the position of research assistant. At this time the first M-6000 and ES computers appear at the institute, which make it possible to produce and explore the first diffractive optical elements (DOE), which at that time were called digital or machine holograms.

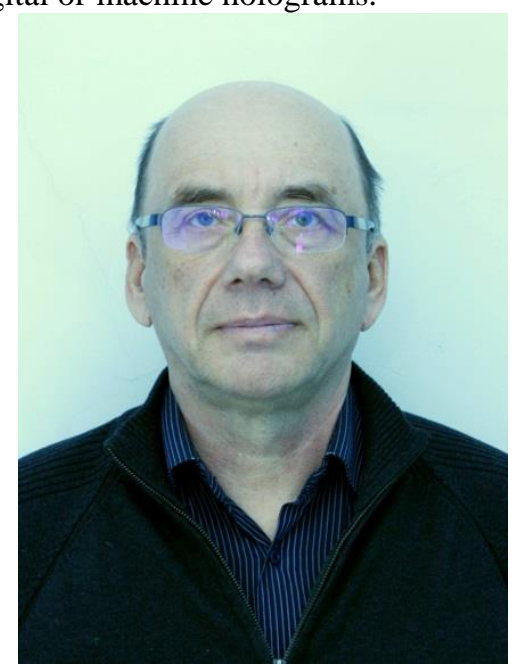

Fig. 1. Doctor of Physical and Mathematical Sciences, Professor S.V. Karpeev

After graduating with honors in 1979 by the direction of the Ministry of Higher and Secondary Specialised Education of the RSFSR Sergey Karpeev began working as an engineer. 1980 became the year when the Soifer group began cooperation with an office of the Physical Institute of the USSR Academy of sciences (later GPI AS), which has had a huge impact on the fate of Sergey Karpeev and on the whole group of Soifer in general. Director of GPI was a Nobel Prize winner Academician A.M. Prokhorov, and the locomotive of this cooperation from the GPI side then was the Head of the laboratory of oscillations I.N. Sissakian, a great scientist and a wonderful person, who died already more than 20 years ago. It was then that the first results of cooperation of mathematicians and programmers with physicists- opticians appeared. Soon emerged an important area of cooperation, which was destined to become one of the breakthrough - focusators of laser radiation. The theme of controlled thermonuclear fusion was then on everyone's lips, and one of the fundamental problems the 
scientists were faced with was control of the area shape of focusing laser radiation, acting on plasma. Then once again the gift for foresight of a great man, Academician A.M. Prokhorov, was confirmed, who offered to carry out laser radiation focusing in specified regions of space with the help of computer synthesized elements which were named focusators. First focusator in longitudinal section [1] was made by Karpeev acccording to calculations of M.A. Golub, and was investigated in the visible wavelength range. This teamwork with A.M. Prokhorov, I.N. Sissakian, V. A.Soyfer and M.A.Golub [1] is the most quoted Karpeev`s work today (65 references), and it continues to be actively cited. At the same time another significant trend was born in computer optics, to which Sergey Karpeev switched. V.A. Soifer started to advance the idea of decomposition of the coherent optical fields into orthogonal components using DOE. The idea which at first seemed abstract gained relevance and physical meaning only with the beginning of joint work with GPI. S.G. Krivoshlykov, who worked at that time in the GPI (now a professor in Boston) and was engaged in fiber optics, helped to formulate precisely the task of measuring the spectrum of transverse modes of optical fiber light guides, for which there was no satisfactory solution with the help of devices known at that time. Using the unique properties of DOE which allowed to implement physically what was set only by a mathematical description brought the solution of the problem to another level. In a short time Karpeev managed to implement appropriate spatial filters, called Modans, and to conduct field experiments with optic fiber light guides [2,3].

All this has helped to create a scientific basis, and in 1983 Karpeev became a correspondence postgraduate in KuAI. His dissertation was planned to be defended in Moscow, in the Council at the GPI on the specialty 01.04.04 "Physical Electronics, including Quantum." According to the Higher Attestation Commission (WAC) regulation at that time only university graduates of physical specialties were allowed to defend their thesis in physical specialties. The rest, including Karpeev, had to pass additional exams in university course of general physics. GPI management regarded this exam and then candidate examination on the specialty very serious, it suffices to say that the examination committee was chaired by N. V.Karlov, the future academician and rector of MIPT. Among the Commission members there also was I.N. Sissakian, Karpeev`s second supervisor, and that further enhances the responsibility. Karpeev has passed both exams successfully, and after that the thesis defense at the most authoritative in this specialty Dissertation Council of the Soviet Union no longer seemed an insurmountable obstacle.

After successfully defending his candidate thesis in 1985 Sergey Karpeev began teaching at KuAI as an assistant of the Department of Technical Cybernetics. It was necessary to introduce a number of new courses for the newly emerging specialization associated with diffractive optics on the faculty. In 1990 Karpeev received the title of associate professor in the Department of Technical Cybernetics, and next 15 years he worked at the department, teaching both subjects for optical information specializations and for future professionals in the field of image processing. He introduced the courses in optical informatics, optical measurement, optical information technologies and systems, optical information processing. Also he was reading courses on information theory, digital signal processing, automated image processing systems, and others. 
Karpeev did not leave his science activities. The theme of Modans developed rapidly, there appeared phase [3] and multi-channel Modans. Two Karpeev works were published $[4,5]$ in top Western journals Optics Communications and Optics \& Lasers in Engineering. The paper [5] was one of the first dedicated to practical implementation of Modans. The developed technologies made it possible to move to the actual use of Modans for excitation modes in lightguides (optical fibers) to transmit information [619]. An important role was played by Karpeev`s cooperation with German scientists from Jena, which began in the GDR, continuing already in the Federal Republic of Germany. This collaboration has allowed access to the upscale optical equipment, which did not yet exist in Russia. During two month trips to Jena in the framework of the Russian-German project DLR new important experimental results were obtained by Karpeev, which became the basis of joint works [9-12, 18]. The work [4] laid the physical foundations of development of innovative fiber optic sensors in the future [20-22], based on the principles of the mode selection.

\section{IPSI RAS}

In 2005, the director of the Institute of Image Processing Systems (IPSI RAS), laureate of the State Prize of Russia in the field of science and technology, V.A. Soifer proposed to transfer Sergey Karpeev to IPSI RAS in order to enhance his scientific research and preparation of his doctoral thesis. The research results obtained in the previous 20 years formed the basis of the monograph written by Karpeev and published by the Radio and Communication publishing house in 2005.

After defending in 2006 his doctoral dissertation Sergey Karpeev did not leave his pedagogical activity and was elected professor of the Department of nanoengineering of SSASU. Already at the new department he published a textbook jointly with S.N. Khonina, based largely on the results obtained in his doctoral thesis.

The work at the new department has expanded the area of Karpeev`s scientific interests and gave him impetus to address such urgent issues as astigmatic modal transformations [23] and the synthesis and study of photonic crystals [24-28]. But the most significant results in this period were obtained by Karpeev in the field of formation of polarization - inhomogeneous beams by methods of diffractive optics [29-39]. It was in this subject that he for the first time was able to publish an article [36] in one of the highly rated magazines Optics Letters (impact factor 3.34). One of the main applications of inhomogeneously polarized beams is sharp focusing, so the study of electromagnetic fields with subwavelength localization by methods of near-field microscopy [40-51] also becomes one of the main Karpeev`s research topics. His publication in Optics Letters [43] has been prepared on this subject.

An important application of DOE becomes their joint use with birefringent crystals [52-59] for forming optical vortices and beams with inhomogeneous polarization.

And finally, new Karpeev works [60-62] are devoted to the important topic of development and testing of space hyperspectrometers. Under Sergey Karpeev`s direction S.V. Alferov has defended his candidate thesis, many students defended their diploma projects and works. Currently under his leadership 2 postgraduate students are working. 


\section{Public acceptance}

S.V. Karpeev has more than 100 scientific publications, 12 patents, he is the author of the monograph. The article [57], published in the Journal of Optics (impact factor 2.059), was recognized as the best article of the week and included in the review of "Optics in 2015".

The successes of S.V. Karpeev in scientific and pedagogical activity were awarded with:

- a regional premium in Science and Technology in 2008 - the Order of the Governor of the Samara region of 28.04.2009 № 119-p;

- in 2013, the Diploma of the Ministry of Education and Science of the Samara region;

— the title of "Honorary Veteran of Labour of SSAU - a SSAU order, on 23.12.2004.

\section{Conclusion}

In conclusion we would like to wish Sergey Karpeev to have strong health, boundless energy, ongoing scientific curiosity, outstanding students, and new creative achievements.

\section{References}

1. Golub MA, Karpeev SV, Prokhorov AM, Sisakyan IN, Soifer VA. Focusing of radiation into a proper space domain with computer-generated holograms. Soviet Technical Physics Letters, 1981; 7(10): 619-626.

2. Golub MA, Karpeev SV, Krivoshlykov SG, Prokhorov AM, Sisakyan IN, Soifer VA. Spatial filter investigation of the distribution of power between transverse modes in a fiber waveguide. Soviet Journal of Quantum Electronics, 1984; 14(9): 1255-1256.

3. Golub MA, Karpeev SV, Kazanskiǐ NL, Mirzov AV, Sisakyan IN, Sowfer VA, Uvarov GV. Spatial phase filters matched to transverse modes. Soviet Journal of Quantum Electronics, 1988; 18(3): 392-393.

4. Garitchev VP, Golub MA, Karpeev SV, Krivoshlykov SG, Petrov NI, Sissakian IN, Soifer VA, Haubenreisser W, Jahn J-U, Willsch R. Experimental investigation of mode coupling in a multimode graded-index fiber, caused by periodic microbends using computer-generated spatial filters. Optics Communication, 1985; 55(6): 403-405.

5. Berezny AE, Karpeev SV, Uspleniev GV. Computer-generated holographic optical elements produced by photolithography. Optics and Lasers in Engineering, 1991; 15(5): 331340.

6. Soifer VA, Karpeev SV, Pavelyev VS, Duparre M, Luedge B. Realisation of an optical interconnection concept using transversal mode selection. Proc. SPIE, 2001; 4316: 152-162.

7. Soifer VA, Pavelyev VS, Karpeev SV Designing DOEs for real-time analysis of the beam mode content. Proc. SPIE, 2001; 4316: 163-169.

8. Karpeev SV, Pavelyev VS, Soifer VA. Designing DOE's for On Line Analysys of Modal Composition. Optical Memory and Neural Networks, 2000; 9 (4): 281-286. 
9. Karpeev SV, Pavelyev VS, Duparre M, Luedge B, Rockstuhl C, Schroeter S. DOE-aided Analysis and Generation of Transverse Coherent Light Modes in a Stepped-Index Optical Fiber. Optical Memory and Neural Networks (Information Optics), 2003; 12 (1); 27-34.

10. Karpeev SV, Pavelyev VS, Soifer VA, Doskolovich L L, Duparre M, Luedge B. Mode multiplexing by diffractive optical elements in optical telecommunication. Proc. SPIE, 2004; 5480: 153-165.

11. Karpeev SV, Pavelyev VS, Soifer VA, Duparre M, Luedge B. Diffractive microrelief design for waveguiding beam control. Proc. SPIE, 2004; 5466 (15): 108-119.

12. Karpeev SV, Pavelyev VS, Soifer VA, Khonina SN, Duparre M, Luedge B, Turunen J. Transverse mode multiplexing by diffractive optical elements. Proc. SPIE, 2005; 5854(01): $1-12$.

13. Karpeev SV, Pavelyev, Kazanskiy NL. Steplike Fiber Modes Excitement with Binary Phase DOEs. Optical Memory \& Neural Networks (Information Optics), 2005; 14(4): 223 228.

14. Borodin SA, Karpeev SV, Kazanskiy NL. Numerical and Experimental Studies of Dispersionless Multimode Beams Generated Using a DOE. Optical Memory \& Neural Networks (Information Optics), 2005; 14(2): 136-141.

15. Borodin SA, Golovashkin DL, Karpeev SV, Kazanskiy NL, Kashin VV. Realization and characterization of diffraction microrelief fabricated on the end faces of halogenide IR waveguide. Optical Memory \& Neural Networks (Information Optics), 2006; 15(3): 135-140.

16. Karpeev SV, Khonina SN. Experimental excitation and detection of angular harmonics in a step-index optical fiber. Optical Memory \& Neural Networks (Information Optics), 2007; 16(4): 295-300.

17. Pavelyev VS, Moiseev OYu, Volkov AV, Eropolov VA, Dmitriev SV, Karpeev SV, Artyushenko VG, Kashin VV. Realization and characterization of diffraction microrelief on the end faces of silver-halide waveguide. Proc. SPIE, 2008; 6994: 69940Q.

18. Gavrilov AV, Karpeev SV, Kazanskiy NL, Pavelyev VS, Duparre M, Luedge B, Schroeter S. Selective excitation of step-index fiber modes. Proc. SPIE, 2007; 6605: 660508.

19. Lyubopytov VS, Tlyavlin AZ, Sultanov AKh, Bagmanov V.Kh. Khonina SN, Karpeev SV, Kazanskiy NL. Mathematical model of completely optical system for detection of mode propagation parameters in an optical fiber with few-mode operation for adaptive compensation of mode coupling. Computer Optics, 2013; 37(3): 352-359.

20. Karpeev SV, Pavelyev VS, Khonina SN, Kazanskiy NL. High-effective fiber sensors based on transversal mode selection. Proc. SPIE, 2005, 5854(16): 163-169.

21. Karpeev SV, Pavelyev VS, Khonina SN, Kazanskiy NL, Gavrilov AV, Eropolov VA. Fibre sensors based on transverse mode selection. Journal of Modern Optics, 2007; 54(6): 833-844. DOI: 10.1080/09500340601066125.

22. Leonovich, GI, Paranin VD, Karpeev SV. Correction of parameters of fiber-optical systems on the basis of the magneto tunable gradient elements. CEUR Workshop Proceedings, 2015; 1490: 133-137. DOI: 10.18287/1613-0073-2015-1490-133-137.

23. Khonina SN, Zoteeva OV, Karpeev SV. Astigmatic transformation of one-dimensional distributions for generation optical vortex arrays. Pacific Science Review, 2011; 13(3): 233-241.

24. Pavelyev VS, Karpeev SV, Dyachenko PN, Miklyaev YV. Fabrication of threedimensional photonics crystals by interference lithography with low light absorption. Journal of Modern Optics, 2009; 56(9): 1133-1136. 
25. Dyachenko PN, Karpeev SV, Pavelyev VS. Fabrication and investigation of threedimensional metallodielectric photonic crystals for infrared range. Computer Optics, 2010; 34(4): 501-505.

26. Dyachenko PN, Karpeev SV, Fesik EV, Miklyaev YV, Pavelyev VS, Malchikov GD. Fabrication of three-dimensional metallodielectric photonics crystals by interference lithography. Proc. SPIE, 2010; 7713: 77131J.

27. Dyachenko PN, Karpeev SV, Fesik EV, Miklyaev YV, Pavelyev VS, Malchikov GD. The three-dimensional photonic crystals coated by gold nanoparticles. Optics Communications, 2011; 284(3): 885-888.

28. Dyachenko PN, Karpeev SV, Pavelyev VS. Fabrication and characterization of threedimensional metallodielectric photonic crystals for infrared spectral region. Optics Communications, 2011; 284(22), 5381-5383.

29. Karpeev, SV, Khonina SN. The optical scheme for universal generation and conversion of nonuniform polarized laser beams by means of DOEs. Computer Optics, 2009; 33(3): 261-267.

30. Karpeev SV, Khonina SN. Simple technique of generation inhomogeneously polarized laser beams by means of DOEs. Computer Optics, 2011; 35(1): 54-62.

31. Khonina SN, Karpeev SV. Grating-based optical scheme for the universal generation of inhomogeneously polarized laser beams. Applied Optics, 2010; 49(10): 1734-1738.

32. Khonina SN, Karpeev SV. DOE-based optical scheme for the universal generation and conversion of inhomogeneously polarized laser beams. Proc. SPIE, 2010; 7523: 75230H.

33. Khonina SN, Karpeev SV. Generating inhomogeneously polarized higher-order laser beams by use of diffractive optical elements. Journal of Optical Society of America A, 2011; 28(10): 2115-2123.

34. Karpeev SV, Khonina SN, Kazanskiy NL, Moiseev OYu. Forming inhomogeneously polarized higher-order laser beams on the basis of circulary polarised beams. Computer Optics, 2011; 35(2): 224-230.

35. Karpeev SV, Khonina SN. Simple technique of generation inhomogeneously polarized laser beams by means of DOEs. Computer Optics, 2011; 35(1): 54-62.

36. Khonina SN, Karpeev SV, Alferov SV. Polarization converter for higher-order laser beams using a single binary diffractive optical element as beam splitter. Optics Letters, 2012; 37(12): 2385-2387.

37. Karpeev SV, Khonina SN, Alferov SV. Generation and conversion of mode beams and their polarization states on the basis of diffractive optical element application. Optical Engineering, 2013; 52(9): 091718-1-091718-8.

38. Paranin, VD, Karpeev SV, Khonina SN. Generation of radially polarized beams based on the refractive optical elements with interference polarizing coatings. Computer Optics, 2015; 39(4): 492-499.

39. Paranin VD, Karpeev SV, Khonina SN. Modeling of forming radially polarized beams on the basis of refractive elements with interference polarizer. Proc. SPIE, 2016; 9807 : $98070 \mathrm{H}$.

40. Karpeev SV, Khonina SN, Kazanskiy NL, Alferov SV. Investigation of focusing inhomogeneously polarized higher-order laser. Computer Optics, 2011; 35(3): 335-338.

41. Karpeev SV, Khonina SN, Alferov SV. Investigation of focusing inhomogeneously polarized higher-order laser beams. Computer Optics, 2012; 36(4): 506-510.

42. Khonina SN, Karpeev SV, Alferov SV, Savelyev DA. Experimental demonstration of generation of longitudinal component of the electric field on the optical axis by high-aperture binary axicon for linear and circular polarization of the incident beam. Computer Optics, 2013; 37(1): 76-87. 
43. Khonina SN, Alferov SV, Karpeev SV. Strengthening the longitudinal component of the sharply focused electric field by means of higher-order laser beams. Optics Letters, 2013; 38(17): 3223-3226.

44. Khonina SN, Alferov SV, Moiseev OYu, Karpeev SV. Study of polarization sensitivity OF near-field microscope using a binary phase plate. Computer Optics, 2013; 37(3): 326-331.

45. Khonina SN, Karpeev SV, Alferov SV, Savelyev DA, Laukkanen J, Turunen J. Experimental demonstration of the generation of the longitudinal E-field component on the optical axis with high-numerical-aperture binary axicons illuminated by linearly and circularly polarized beams. Journal of Optics, 2013; 15(8): 5704 (9 pp).

46. Alferov SV, Karpeev SV, Khonina SN, Tukmakov KN, Moiseev OYu, Shulyapov SA, Ivanov KA, Savel'ev-Trofimov AB. On the possibility of controlling laser ablation by tightly focused femtosecond radiation. Quantum Electronics, 2014; 44(11): 1061-1065.

47. Alferov SV, Khonina SN, Karpeev SV, Savelyev DA. Experimental generation of the longitudinal electric field component on the optical axis with high-numerical-aperture binary axicons. Proceedings of SPIE, 2014; 9533: 95330D.

48. Degtyarev SA, Khonina SN, Alferov SV, Karpeev SV. Theoretical and experimental study of aperture size effects on the polarization sensitivity of near-field microscopy fiber-optic probes. Proceedings of SPIE, 2014; 9156: 915608.

49. Alferov SV, Khonina SN, Karpeev SV. Study of polarization properties of fiber-optics probes with use of a binary phase plate. Journal of Optical Society of America A, 2014; 31(4): 802-807.

50. Alferov SV, Karpeev SV, Khonina SN, Moiseev OYu. Experimental study of focusing of inhomogeneously polarized beams generated using sector polarizing plates. Computer Optics, 2014; 38(1): 57-64.

51. Zayarny DA, Ionin AA, Kudryashov SI, Makarov SV, Rudenko AA, Bezhanov SG, Uryupin SA, Kanavin AP, Emel'yanov VI, Alferov SV, Khonina SN, Karpeev SV, Kuchmizhak AA, Vitrik OB, and Kulchin YuN. Nanoscale boiling during single-shot femtosecond laser ablation of thin gold films. JETP Letters, 2015; 101(6): 394-397.

52. Khonina SN, Karpeev SV, Alferov SV. Theoretical and an experimental research of polarizing transformations in uniaxial crystals for generation cylindrical vector beams of high orders. Computer Optics, 2014; 38(2): 171-180.

53. Khonina SN, Morozov AA, Karpeev SV. Effective transformation of a zero-order Bessel beam into a second-order vortex beam using a uniaxial crystal. Laser Physics, 2014; 24(5): 056101-056105.

54. Khonina SN, Paranin VD, Karpeev SV, Morozov AA. Study of polarization transformations and interaction of ordinary and extraordinary beams in nonparaxial regime. Computer Optics, 2014; 38(4): 598-605.

55. Paranin VD, Karpeev SV, Krasnov AP. A converter of circularly polarized laser beams into cylindrical vector beams based on anisotropic crystals. Computer Optics, 2015; 39(5): 644-653. DOI: 10.18287/0134-2452-2015-39-5-644-653.

56. Paranin VD, Karpeev SV. Method of measurement of thickness of uniaxial anisotropic crystals and thermal control of bessel beam transformation. Computer Optics, 2016; 40(1): 36-44. DOI: 10.18287/0134-2452-2016-40-1-36-44.

57. Khonina SN, Karpeev SV, Alferov SV, Soifer VA. Generation of cylindrical vector beams of high orders using uniaxial crystals. Journal of Optics, 2015; 17: 065001-11 pp.

58. Paranin VD, Karpeev SV, Khonina SN. Control of the formation of vortex Bessel beams in uniaxial crystals by varying the beam divergence. Quantum Electronics, 2016. 46(2). P. 163-168. 
59. Paranin VD, Karpeev SV, Kazanskiy NL, Krasnov AP. Converter of laser beams with circular polarization to cylindrical vector beams based on anisotropic crystals. Proc. SPIE, 2016; 9807: 98070R.

60. Karpeev SV, Alferov SV, Khonina SN, Kudryashov SI. Study of the broadband radiation intensity distribution formed by diffractive optical elements. Computer Optics, 2014; 38(4): 689-694.

61. Karpeev SV, Ustinov AV, Khonina SN. Design and analysis of a three-wave diffraction focusing doublet. Computer Optics, 2016; 40(2): 173-178. DOI: 10.18287/0134-24522016-40-2-173-178.

62. Karpeev SV, Khonina SN, Kharitonov SI. Study of the diffraction grating on the convex surface as a dispersive element. Computer Optics, 2015; 39(2): 211-217. DOI: 10.18287/01342452-2015-39-2-211-217. 